\title{
Analysis on Risk Decision and Avoidance strategy based on the Bayesian Formula
}

\author{
Xiaoying Wang ${ }^{1, a}$ \\ ${ }^{1}$ ChifFeng University, Chifeng, 024000, China \\ a527514533@qq.com
}

Keywords: Bayesian formula; Risk decision; Mitigation strategy

\begin{abstract}
In the investment decision, we often come across the decision problem in an uncertain state operating, for this kind of problem solely on subjective or objective data for decision making, the blindness and risk are larger. Therefore, in the increasingly fierce competition of the market economy condition, need to have a scientific approach to investment decision to provide smaller risk solutions. If some sort of sampling test before making decisions, the information provided by decision maker according to the result of sampling, on the state of nature that affect the decision of increase understanding and then make decisions, which can improve the correctness of decisions. This research through the instance, the risk decision are discussed in detail how to use the Bayesian formula in effectively integrate relevant information, choose the optimal strategy, and explain the optimal decision.
\end{abstract}

\section{Introduction}

The so-called decision-making is decision makers may be met in order to solve the current or future problems, in a number of alternative action plan, select a best solution process in some sense. Decisions correctly or not will bring enterprise gains or losses. Therefore, policy makers should learn to reasonable decision analysis, to avoid heavy losses. Because there are a lot of uncertain factors in the decision environment and inadequate statistics, decision inevitably has some degree of risk. The powerful tool for the available information is to reduce the risk. In general, the more fully, the uncertainty of decision-making environment is smaller, the smaller the risk. Bayesian statistical method the basic idea is to make full use of the model information (assumption), the mathematical model, data information (sampling information) and a priori information (experience), the prior distribution and integration into the posterior distribution, sampling distribution test after the starting point of distribution for decision making. If there is new information (data), then update the posterior distribution, achieve recursive decision-making plan. Because there are lots of uncertain factors in the market environment and policy makers it limits of knowledge ability, coupled with inadequate statistics, decision makers often unable to grasp all the information related to decision-making, and makes the decision of the subjective and objective facts or deviation between the targets. So under the condition of incomplete information of decision will inevitably bring some degree of risk to decision makers. Information is a powerful means to reduce the risk. The more fully, the uncertainty of decision-making environment is smaller, the smaller the risk. But no matter use what means to collect information, require a certain input, it takes the necessary manpower and material resources, financial resources and time; this is the cost price for access to information, the information cost. To master a large number of high-quality information, can improve the reliability of decision-making, and, in turn, increase the economic benefit, it embodies the value of information.

\section{The risk decision theory basis}

The risk decision concept. The basic meaning of risk is uncertainty of loss. However, for the basic starting point and the focus of the concept vary, it also each is not identical in different definition of the human eye. Because this article mainly discusses the risk decision problem, therefore, the risk is defined as the uncertainty. And this uncertainty can be divided into the 
uncertainty of objective and subjective uncertainty. Objective uncertainty is actual results and expected results of deviation; it can use statistical tools to measure them. Subjective uncertainty is a person of objective risk assessment, it with personal knowledge, experience, mental and psychological state, the same objective risk faced by different people will have different subjective uncertainty. The risk for each unit of risk are complicated, and deal with the risk of a particular method and can be used a variety of. To meet with the minimum input for maximum security goal, must choose the best combination of all the countermeasures, which is the important content in the process of risk decision. Risk decision of preparation works for decision-making to provide the necessary information and decision-making basis, to help make risk management personnel as far as possible the risk of scientific and reasonable decision.

The meaning and principles of risk decision-making. A key role in decision-making work in risk management can be reflected in the decision itself connotation: first, the risk decision depends on the risk management objective, risk decision corresponds to the risk management goal, is the basis and to realize the objective of risk management, security, must ensure that risk decision taken by the can achieve with minimum cost to obtain the biggest security management goals. Second, the risk decision is the optimization of various risk management methods and the integrated use of, from the macroscopic Angle to formulate action plan as a whole. Develop risk management plan should be based on risk management objectives, analysis of risk factors, risk level, understand the pros and cons of alternative methods and costs, make reasonable choice and the combination after comprehensive evaluation. At the same time, the risk itself has some characteristics, such as objective existence, contingency and variability, make the risk decision has the characteristics of different from other general management decisions. In order to guarantee the realization of the objective of risk management, risk management decision should insist on the principle of comprehensive, strengths, cost-benefit comparison principle and pay attention to the use of commercial insurance, but don't ignore the principles of other methods.

\section{The Bayesian decision model}

Every risk decision-making problem includes three elements: the state of nature (natural state formation), decision makers to take action (action), decision makers to take the consequences of an action (gain or loss function description). Starting from the three elements, can be different risk scenario space. In usually decision making problems, the decision makers in nature (or social) accumulates a lot of experience and data, the priori information is not enough to determine the nature of what would happen (or social) state, but in many occasions to the state set on a prior distribution is given. Learned about the probability estimates of various states appeared. The a priori information can be used when making decisions, which based on the prior probability distribution and expectation criterion for the choice of the optimal solution. Because of the prior probability has strong subjective color, can not fully reflect the objective laws, in order to make decisions, it is necessary to further supplement the new information, new data, thus correct prior probability and get a posteriori probability. Posterior probability is calculated according to the Bayesian formula in probability theory, so called the decision-making Bayesian decision model.

Bayesian decision analysis is under incomplete information, for some unknown state with subjective probability estimates, and then used to modify probability Bayesian formula, finally re-uses expectations and fixed probability to make optimal decisions. Bayesian decision theory is a basic method of statistical model of decision. The basic idea is: (1) the known with the unknown parameters of probability density expression as well as the unknown parameters of the prior probability; (2) the prior distribution is used to calculate by probabilistic; (3) according to the parameters of the Bayesian posterior probability o decisions. Seek the Bayesian decision function has two paths, one is minimize the risk of posterior, one was to make the minimum risk. Bayesian analysis model and calculation method is as follows: 
As $A_{1}, A_{2}, \cdots A_{n}$ is a complete event group, event only in the complete event group $\mathrm{B}$ a certain event occurs, and $P(B)>0$, under the condition of event $\mathrm{B}$ occurs, the conditional probability of event $A_{i}$ is $P\left(A_{i} / B\right)$, It is calculated by the following Bayesian formula:

$$
P\left(A_{i} / B\right)=\frac{P\left(A_{i}\right) P\left(B / A_{i}\right)}{P(B)}
$$

On the type of $P\left(A_{i} / B\right)$ said conditional probability under the condition $A_{i}$ of incident happen, $P(B)$ is the event $\mathrm{B}$ of full probability, that:

$$
P(B)=\sum_{i=1}^{n} P\left(A_{i}\right) P\left(B / A_{i}\right)
$$

For Bayesian decision, first prior analysis, is the detailed list decision matrix or the decision tree, the result of the incidence of the various states and conditions are to estimate or calculate, but if people could further gathering information, it is possible to make the decision for more sure. Is so-called decision tree, the organization and said policymakers decisions and uncertain problems faced by a systematic way, by means of an uncertain event a weighted average of all possible numerical results of calculation, it is concluded that the expected currency value, thus according to the merit principle, evaluating and decision-making method of choice. This article also Bayesian analysis reflected in the process of decision tree analysis, for the application and research of uncertainty risk decision.

\section{The specific application of Bayesian formula in risk decision}

To ask questions. A particular set of homes for sale price is $\$ 400000$, and listed only one day. Have A customer. A going to buy the house, his agent B that saw this house in the day of the customer, at least 20 or more customers want to buy this house. B added that another agent to discuss matters relating to the purchase of this house that afternoon. B advice if you decide to buy, bid price should be close to $\$ 400000$. If another purchase prices of the similar competitors out of this house, the seller usually require customers to buy house the next day to give the final price.

For this situation, customers think a house can consider to $\$ 400000$. If he buy this house is successful, he gave the "emotional value assignment of this house for $\$ 10000$. That is to say, if acceptable market price is $\$ 400000$, he needs to spend $\$ 410000$ to buy this house. So, if he succeeded in buying this house for $\$ 390000$, the house finally gains the result value of $\$ 20000$. Of course, if they did not succeed to buy this house, so the results of this house value will only be $\$ 0$. A customer for he is only A competition of the probability of the participants also assign A value of 0.3 .

He has decided to consider choosing the following one of the three kinds of price: $\$ 390000$, $\$ 400000$ or $\$ 405000$. He estimates that if he is only a competition of participants, so the probability of price of $\$ 390000$ is 0.40 , cost 400000 Yuan price of probability is 0.60 , the probability of price of $\$ 405000$ is 0.90 . However, if there are other competitors, A hypothesis seller asked him to submit final price the next day. In this case, he will have to reconsider to make a decision: he can cancel the purchase plan, with the same purchase plan, or add another $\$ 5000$ purchase plan. He thinks that in the presence of many competitors, the probability of price of $\$ 390000$ is 0.20 , the probability of price of $\$ 395000$ is 0.30 , the probability of price of $\$ 400000$ is 0.50 , the probability of price of $\$ 405000$ is 0.70 , and the probability of $\$ 410000$ price is 0.80 .

To solve the problem. Objective to make decision to buy A house, in order to consider customers A choice using Bayesian formula decision-making method, to predict the results of the different decision, and to build A decision tree model to help him make the decision. Can be according to the front of the train of thought for decision analysis, Bayesian analysis result is reflected in the decision tree model.

By the decision tree analysis method, which can be clearly seen in the face of each kind of uncertainty, the need to make risk decision, which are given a probability, the possibility of 
according to the different values and the result is different, and can be further using Bayesian decision-making method, the results of different events and the combination of probability of occurrence, corresponding with different risk decision results brought about by the weighted average, according to the results, to decisions have a clear understanding of and to have a preliminary decision event value evaluation, has considerable reference significance. To illustrate ideas in the process of decision tree to solve, choose to offer customers A $\$ 390000$ decision analysis: in this case, risk decision events as the "purchase price decision problem", taken by the method of " Bayesian decision", will be the result of the uncertainty clarity as far as possible, to reduce loss to minimum, in order to benefit maximization.

The case analysis from the above we know, engaged in economic activities, before the decision about the state of random factors in advance can get the more information, more reliable, the more reliable, accordingly to make the optimal decision of the expected benefits value may also be higher. On the other hand, if the less access to information, the more unreliable, then get the reliability of the optimal decision of the poor, expected benefit value may also be lower. And choose suitable risk decision-making method according to the circumstance, also can let you according to the lateral reach twice the result with half the effort. First you need to make market survey; secondly organize production according to the result of investigation: when it has a large market research conclusion to make mass production, when the conclusion is demand for hours to small batch production, so the biggest profit. Through the above case analysis can get, in the process of decision making under risk, careful market investigation, using the Bayesian formula prior probability can be modified for the a posteriori probability, and then calculate a posteriori expected return, choose the optimal risk strategy. This strategy is very useful in practice.

\section{Summary}

Risk decision-making scheme selection depends on the external environment condition, and this state is uncertain, more is not controlled by policy makers, but by judgment, investigation and experiment can obtain relevant information. The use of statistical decision method can work out a reasonable risk investment decisions. Bayesian decision belongs to the risk type decision, decision makers are unable to control the change of the objective factors, but be able to handle the change may state and the distribution probability of each state, and use the average expectation that the future possible conditions as decision criteria. Due to policy makers on the description of the objective factors change in uncertain, so in their decision-making for policy makers pose a risk. But entirely sure in reality almost does not exist, the Bayesian decision not to make decision problem entirely risk-free, but through other ways to increase the amount of information to make decision of risk reduction. Thus it can be seen that Bayesian decision making is a kind of more practical ways.

\section{References}

[1] Han Lu,Han Liyan,Zhao Hongwei. Credit Scoring Model Hybridizing Artificial Intelligence with Logistic Regression. Journal of Networks. 2013 (1).

[2] Ling-Jing Kao,Chih-Chou Chiu,Fon-Yu Chiu. A Bayesian latent variable model with classification and regression tree approach for behavior and credit scoring. Knowledge-Based Systems. 2012(06).

[3] Stjepan Oreski,Dijana Oreski,Goran Oreski. Hybrid system with genetic algorithm and artificial neural networks and its application to retail credit risk assessment. Expert Systems With Applications. 2012 (16).

[4] Wu Jee Cheng,Lin Bo Kai,Tsuei Gwo Chyang. Comparison of Processing Chains Based on Support Vector Machine Classifier for Hyperspectral Image Classification. Advanced Materials Research. 2012 (433). 
[5] Paulius Danenas, Gintautas Garsva,Saulius Gudas. Credit Risk Evaluation Model Development Using Support Vector Based Classifiers. Procedia Computer Science. 2012(10).

[6] Defu Zhang,Xiyue Zhou,Stephen C.H. Leung,Jiemin Zheng. Vertical bagging decision trees model for credit scoring. Expert Systems With Applications. 2010 (12). 